\title{
EXPECTED SCIENTIFIC PERFORMANCE OF THE THREE SPECTROMETERS ON THE EXTREME ULTRAVIOLET EXPLORER
}

\author{
J. V. Vallerga , P. Jelinsky, P. W. Vedder, and R. F. Malina \\ Space Sciences Laboratory, University of California, Berkeley, CA, USA
}

\begin{abstract}
The expected in-orbit performance of the three spectrometers included on the Extreme Ultraviolet Explorer (EUVE) astronomical satellite is presented. Recent calibrations of the gratings, mirrors and detectors using monochromatic and continuum EUV light sources allow the calculation of the spectral resolution and throughput of the instrument. An effective area range of 0.2 to $2.8 \mathrm{~cm}^{2}$ is achieved over the wavelength range $70-600 \AA$ with a peak spectral resolution $\lambda / \Delta \lambda$ (FWHM) of -360 assuming a spacecraft pointing knowledge of 10 arc seconds (FWHM). For a $40,000 \mathrm{sec}$ observation, the average $3 \sigma$ sensitivity to a monochromatic line source is $3 \times 10^{-3}$ photons $\mathrm{cm}^{-2} \mathrm{sec}^{-1}$. Simulated observations of known classes of EUV sources such as hot white dwarfs and cataclysmic variables are also presented.
\end{abstract}

\section{INTRODUCTION}

During the first six-month phase of its mission, EUVE will carry out an all-sky survey in the EUV ( $70 \AA$ to $750 \AA$ ) in four photometric bandpasses defined by thin film filters, as well as a deep survey along the ecliptic (Bowyer 1983). The second phase of the mission is devoted to the spectrometer with long pointings (typically $40,000 \mathrm{sec}$ ) at targets selected by guest observers. The calculations made in this paper are based on calibration results of flight hardware components (Jelinsky et al. 1988). These components are to be integrated in 1989, and launch is set for September, 1991, on a Delta II rocket.

\section{INSTRUMENT DESCRIPTION}

The EUVE spectrometers consist of three varied line-spaced plane reflection gratings mounted in the converging beam behind a single grazing incidence Wolter-Schwarzschild type Il telescope (Hettrick et al. 1985). Each grating has a dedicated microchannel plate (MCP) detector (Siegmund et al. 1986), thus providing simultaneous coverage of an EUV source in

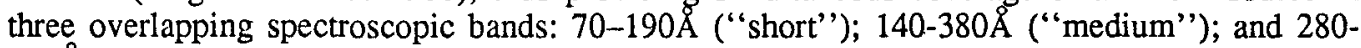
$760 \AA$ ("long"). Each detector has a set of thin film filters, which act as order filters and attenuate any scattered Ly $\alpha$, the dominant component of nighttime airglow with an intensity of 3500 Rayleighs (Chakrabarti 1984). The other major airglow lines at $304 \AA$ and $584 \AA$, caused by resonance scattering of solar flux by $\mathrm{He}$ II and $\mathrm{He}$ I, fall withing the spectroscopic bandpasses and require the inclusion of a wire grid collimator in the medium and long wavelength channels to limit this diffuse flux to a small part of the spectrum.

The effective area of the three spectrometer channels is shown in Fig 1. This includes the geometrical area (1/6 of telescope area), mirror reflectivity, grating efficiency, order filter transmission, and detector quantum efficiency. Also shown is the spectral resolution, $\lambda / \Delta \lambda$ (FWHM), of each channel, which is limited primarily by the aberrations of the gratings. Given these characteristics, a diffuse background model (Chakrabarti, Kimble, and Bowyer 1984), and an integration time, we can calculate a minimum detectable line flux (photons $\mathrm{cm}^{-2} \mathrm{sec}^{-1}$ ) vs. wavelength, which is shown in Fig 2 . Note the decrease in sensitivity around $304 \AA$ and $584 \AA$ due to the diffuse airglow. Observations in the Earth's shadow are more sensitive near $304 \AA$ than those shown in Fig. 2, but the $584 \AA$ sensitivity remains the same since most of the $584 \AA$ flux is interplanetary in origin. 


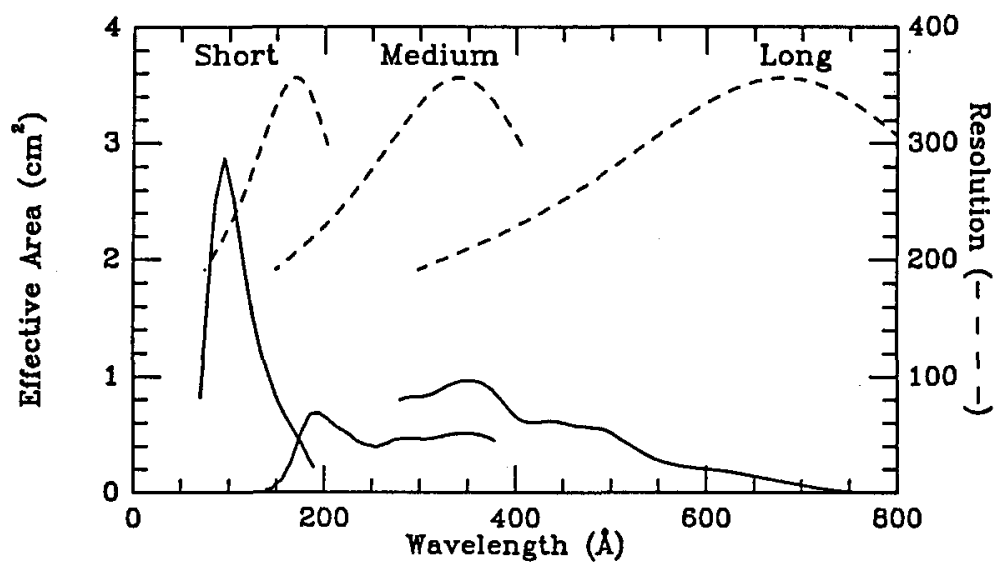

Figure 1. EUVE Spectrometer Characteristics. The plotted effective area (solid line, left scale) for the three spectrometer channels combines the geometric area with the measured throughput of the individual components in each channel (mirror, grating, filter, detector). The calculated resolution $\mathbf{R}$ (= $\lambda \Delta \lambda$ FWHM -- dashed line, right scale) for the three channels assumes spacecraft pointing knowledge of $10^{\prime \prime}(3 \sigma)$ and detector spatial resolution of 70 microns FWHM.

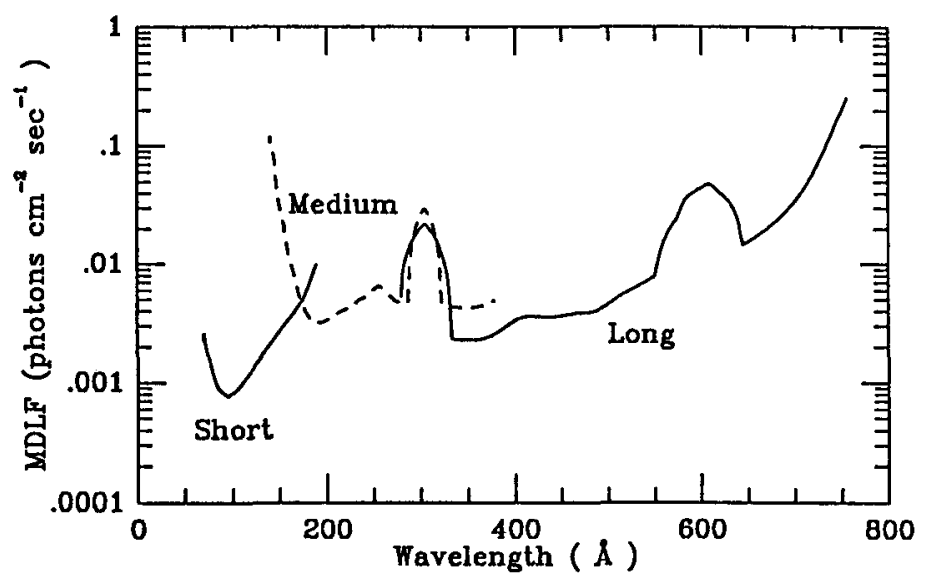

Figure 2. Minimum Detectable Line Flux. Plotted is the minimum flux from an unresolved line in order to be detected at the $5 \sigma$ level above background in a 20,000 second observation for the three spectrometer channels. The wide features centered at $304 \AA$ and $584 \AA$ are due to the partially collimated diffuse background lines for observations away from the Earth's shadow. The MDLF would be lower by a factor of $\approx 6$ at $304 \AA$ in the Earth's shadow, but the sensitivity at $584 \AA$ would remain unchanged since the neutral helium which scatters the solar line is interplanetary. 


\section{SPECTROMETER SIMULATION METHOD}

To simulate the count rate spectra of known classes of EUV sources, we take modelled spectra of EUV sources, calculate the flux for some assumed distance, and attenuate the flux assuming a column density of hydrogen $\left(\mathrm{N}_{H I}\right)$ and a model of the interstellar medium, or ISM (Cruddace et al. 1974). The spectra are then weighted by the effective area (including second and third order diffraction) and convolved with the mirror point spread function, telescope aspect, grating resolution, and detector spatial resolution. Monochromatic diffuse background (airglow) is added convolved with the collimator response, and a uniform detector background count rate is also added. The resultant counts/bin are then randomly distributed based on Poisson statistics. The resultant "raw" count rate spectrum does not include the effects of nonuniform detector response (flat field), image distortion, and scattering, all of which are expected to affect the counts in any bin by no more than $5 \%$.

\section{SIMULATION RESULTS OF EUV SOURCE SPECTRA}

We have simulated two classes of known EUV sources, hot white dwarfs and cataclysmic variables, to show the capabilities of the EUVE spectrometer in observing continuum sources and spectral line sources, respectively. For the hot white dwarf we have taken a model atmosphere (Malina et al. 1982) at 55,000 $\mathrm{K}$ with a small fraction of helium $\left(1 \times 10^{-5}\right)$ and placed it at the distance $(49 \mathrm{pc})$ of the known EUV source G191-B2B with a neutral hydrogen column of $\mathrm{N}_{H I}=8 \times 10^{17} \mathrm{~cm}^{-2}$ (Green, Jelinsky, and Bowyer 1988). Figure 3 shows the resulting raw count spectrum in all three channels. Note the edge due to the stellar He II at $228 \AA$ and at $456 \AA$ ( $228 \AA$ in second order). The strong edge at $504 \AA$ is due to neutral helium in the ISM with an assumed column density equal to $10 \%$ that of neutral hydrogen. White dwarf spectra will serve as sensitive probes of the ISM, as well as provide vital information about the last stages of stellar evolution.

EXOSAT observations of the SU Uma cataclysmic variables VW Hyi and OY Car in superoutburst imply the presence of an extended hot, optically thin corona with temperatures between $10^{6}$ and $10^{7} \mathrm{~K}$ (Van Der Woerd 1987). Spectra of these hot corona in the EUV are very strong functions of temperature, and the EUVE spectrometers can do much to pin down the physics of the emitting region. Figure 4 shows a simulation in the medium wavelength

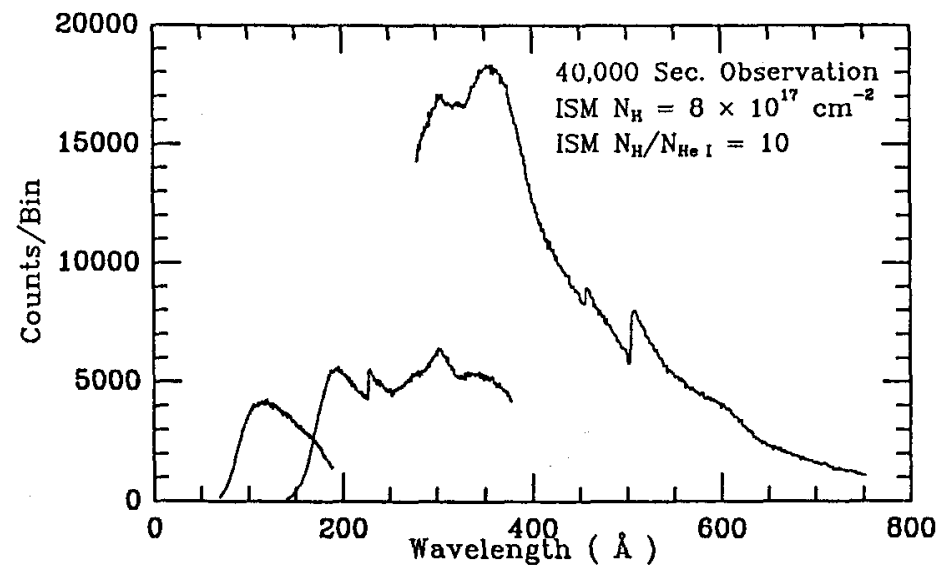

Figure 3. Hot White Dwarf G191-B2B. The simulated raw count spectrum of known EUV source G191-B2B in the three spectrometer channels plotted together. Note the strong detection of the interstellar medium absorption edge of $\mathrm{He}$ I at $504 \AA$ and the white dwarf He II edge at $228 \AA$. 


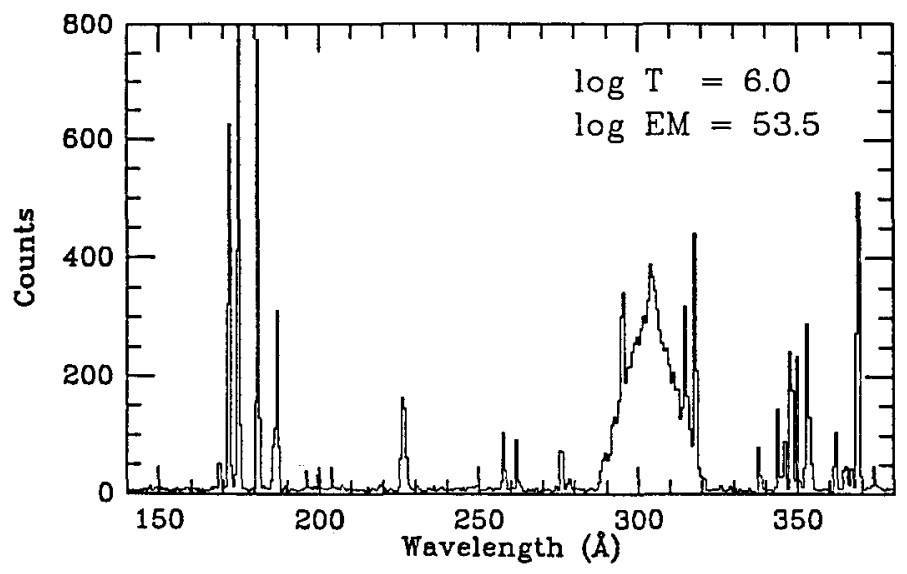

Figure 4. Cataclysmic Variables. Plotted is a medium wavelength channel simulation of a 20,000 second raw count spectrum of a cataclysmic variable in superoutburst with a hot, optically thin corona. The CV was placed at $50 \mathrm{pc}$ with a hydrogen column of $10^{19} \mathrm{~cm}^{-2}$. The $304 \AA$ diffuse background line shows the triangular response of the wire grid collimators.

spectrometer of a coronal spectrum based on the Raymond and Smith code (Raymond and Smith 1977) at $10^{6} \mathrm{~K}$ with an emission measure of $10^{53.5}$ (derived from the EXOSAT data, Van Der Woerd 1987) placed at $50 \mathrm{pc}$ and an ISM column of $\mathrm{N}_{H I}=10^{19} \mathrm{~cm}^{-2}$ and $\mathrm{N}_{H I} / \mathrm{N}_{H e l}=$ 10. The large triangular-shaped bump centered at $304 \AA$ is diffuse airglow through the collimator. The strong emission near $175 \AA$ is a complex of Fe X, Fe IX, and Fe XI lines. Similar spectra can be generated using the coronal model for less exotic but much more abundant and closer late type stars such as M dwarfs.

\section{ACKNOWLEDGEMENTS}

This work was supported by NASA contract NAS5-29298.

\section{REFERENCES}

Bowyer, S. 1983, Adv. Space Res., 2, 157.

Cruddace, R., Paresce, F., Bowyer, S., and Lampton, M. 1974, Ap. J., 187, 497.

Chakrabarti, S., Kimble, R., and Bowyer, S. 1984, J. Geophys. Res., 89, A7.

Green, J., Jelinsky, P., and Bowyer S. 1988, in Proc. Symposium on Cosmic Abundances of Matter, in press.

Hettrick, M. C., Bowyer S., Malina, R. F., Martin, C., and Mrowka S. 1985, Appl. Opt., 24, 1737.

Jelinsky, P., Jelinsky, S. R., Miller, A., Vallerga, J., and Malina, R. F. 1988, Proc. SPIE, 628, in press.

Malina, R. F., Bowyer, S., and Basri, G. 1982, Ap. J., 262, 717.

Raymond, J., and Smith, B. 1977, Ap. J. Suppl., 35, 419.

Siegmund, O. H. W., Lampton, M. L., Chakrabarti, S., Vallerga, J. V., Bowyer S., and Malina, R. F. 1986, Proc. SPIE, 627, 660.

Van Der Woerd, H. 1987, Astrop. Space Sci., 130, 225. 